\title{
Adaptive Reweighting of Auditory Localization Cues in Response to Chronic Unilateral Earplugging in Humans
}

\author{
Daniel P. Kumpik, Oliver Kacelnik, and Andrew J. King \\ Department of Physiology, Anatomy, and Genetics, University of Oxford, Oxford OX1 3PT, United Kingdom
}

Localizing a sound source involves the detection and integration of various spatial cues present in the sound waves at each ear. Previous studies indicate that the brain circuits underlying sound localization are calibrated by experience of the cues available to each individual. Plasticity in spatial hearing is most pronounced during development but can also be demonstrated during adulthood under certain circumstances. Investigations into whether adult humans can adjust to reduced input in one ear and learn a new correspondence between interaural differences cues and directions in space have produced conflicting results. Here we show that humans of both sexes can relearn to localize broadband sounds with a flat spectrum in the horizontal plane after altering the spatial cues available by plugging one ear. In subjects who received daily training, localization accuracy progressively shifted back toward their pre-plug performance after 1 week of earplugging, whereas no improvement was seen if all trials were performed on the same day. However, localization performance did not improve on a task that used stimuli in which the source spectrum was randomized from trial to trial, indicating that monaural spectral cues are needed for plasticity. We also characterized the effects of the earplug on sensitivity to interaural time and level differences and found no clear evidence for adaptation to these cues as the free-field localization performance improved. These findings suggest that the mature auditory system can accommodate abnormal inputs and maintain a stable spatial percept by reweighting different cues according to how informative they are.

\section{Introduction}

To pinpoint the location of a sound source, the auditory system makes use of various cues generated by the interaction between sound waves and the head, external ears, and torso. The separation of the ears on either side of the head gives rise to interaural level differences (ILDs) and interaural time differences (ITDs), which enable sounds to be localized in the horizontal plane, whereas spectral pinna cues support elevation judgments and front-back discrimination (Wightman and Kistler, 1993; Blauert, 1997; King et al., 2001). Each of these cues is potentially ambiguous, and their contributions to spatial hearing depend on the nature of the sound as well as the region of space from which it originates. To maintain a stable representation of space, the auditory system must therefore integrate different cues whose availability and reliability can change over time.

Auditory spatial cue values change during development as the body grows (Clifton et al., 1988; Campbell et al., 2008) and vary in value between individuals according to the physical dimensions of the head and external ears (Shaw and Teranishi, 1968; Middlebrooks and Green, 1990; Xu and Middlebrooks, 2000; Schnupp et al., 2003). Accordingly, there is evidence that

Received Nov. 5, 2009; revised Feb. 22, 2010; accepted Feb. 27, 2010.

This study was supported by the Wellcome Trust through a Principal Research Fellowship (A.J.K.) and a Prize Studentship (0.K.). We are grateful to Joshua Fox for assistance with some of the experiments and to Peter Keating and Ruth Litovsky for valuable discussion. We also thank Ruth Litovsky for suggestions regarding the design of the closed-field response interface and Kim Schairer for advice on bone conduction of sound.

Correspondence should be addressed to Dr. Andrew J. King, Department of Physiology, Anatomy, and Genetics, Sherrington Building, University of Oxford, Parks Road, 0xford 0X13PT, UK. E-mail: andrew.king@dpag.ox.ac.uk. DOI:10.1523/JNEUROSCI.5488-09.2010

Copyright $\odot 2010$ the authors $\quad 0270-6474 / 10 / 304883-12 \$ 15.00 / 0$ humans (Wenzel et al., 1993; Middlebrooks, 1999) and animals (Knudsen et al., 1984; King et al., 2000) learn by experience to localize with their own ears. Although this plasticity is particularly important during the phase of head growth, it is clear that the mature mammalian brain can also relearn to localize sound in the presence of substantially altered auditory spatial cues. For example, adult humans can learn to use altered spectral cues for localization in the vertical plane (Hofman et al., 1998; Van Wanrooij and Van Opstal, 2005), whereas varying degrees of plasticity have been reported after modifying the available binaural cues (Held, 1955; Bauer et al., 1966; Florentine, 1976; Butler, 1987; Javer and Schwarz, 1995; McPartland et al., 1997; Hofman et al., 2002).

Previous studies of auditory spatial learning in adulthood have focused on the extent to which the processing of individual localization cues can change with experience, without addressing whether learning depends on changes in the way in which different cues are integrated within the brain. One approach to investigating this is to alter the spatial cues available by occluding one ear with an earplug. Using this paradigm, Kacelnik et al. (2006) demonstrated that adult ferrets can rapidly relearn to localize sounds accurately and proposed that this plasticity is based on a reweighting of different cues. Here we extend those findings by showing that humans are equally capable of accommodating the altered spatial cues produced by plugging one ear. By showing that learning with altered spatial cues depends on the availability of a reliable source spectrum but is not accompanied by compensatory changes in ILD or ITD processing, our results suggest that the mature auditory system can change the weighting of different cues to maintain a stable percept of auditory space. 


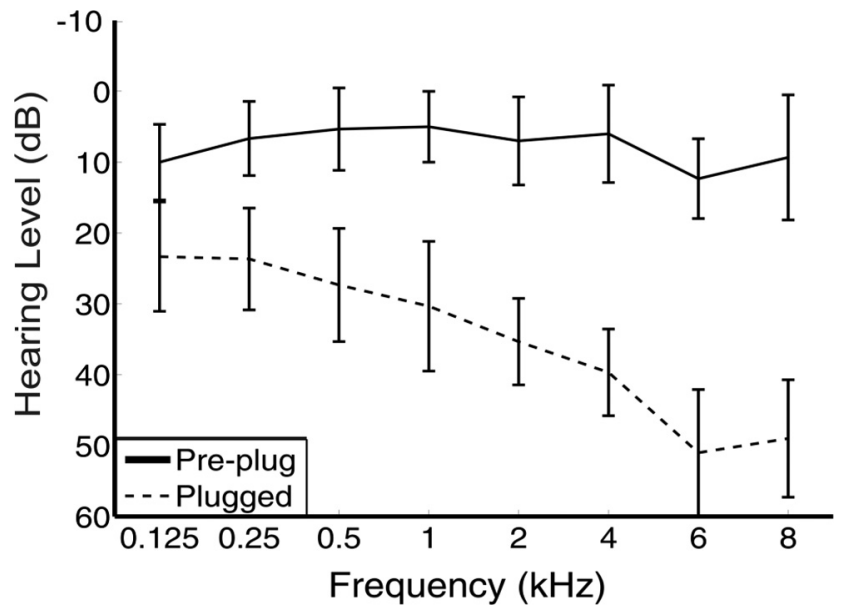

Figure 1. Impact of earplug insertion on audiograms taken from subjects before plugging and after insertion of the earplug. Data are shown for the plugged ear only. Error bars are SDs. The attenuation caused by the earplug is frequency dependent.

\section{Materials and Methods}

Subjects. A total of 26 subjects took part in the experiment to investigate the effects of unilateral earplugging on free-field sound localization of flat noise stimuli. Of these, six were excluded after failing to meet the pretraining performance criteria, as described below. Twenty subjects ( 7 male, 13 female; mean age, 23 years) therefore completed the experiment. These subjects formed three groups: the first group ("blocktrained"; $n=5$ ) performed training with the flat noise stimulus in the presence of an earplug but received all their training in $1 \mathrm{~d}$ to establish whether block exposure to our training task was sufficient to induce improvements in localization. As a comparison with this group, a second group ("daily-trained/flat"; $n=5$ ) performed a similar number of flat noise trials, but these were spread out in blocks of 125 trials per day over $7-8 \mathrm{~d}$. The third group ("daily-trained/all": $n=10$ ) received $8 \mathrm{~d}$ of training with the flat noise stimuli and also performed a larger number of trials (250 per day), in an attempt to determine whether increasing the number of trials could increase the rate at which localization performance improved. This group was also assessed for localization performance using free-field stimuli with a "random-filtered" spectrum before, during, and after the plugged period. For a subset of these subjects $(n=$ 5), we also characterized closed-field binaural sensitivity before, during, and after the plugged period.

All subjects were assessed before testing to ensure normal bilateral audiometric thresholds (i.e., within $20 \mathrm{~dB}$ of normal, from $125 \mathrm{~Hz}$ to 8 $\mathrm{kHz}$ ). Subjects were randomly selected to wear an earplug in either the left or the right ear, with nine subjects falling into the former group. Five subjects had previous experience of psychometric testing, although all participants received extensive pretraining on the relevant localization/ lateralization tasks before the plugging phase began, to ensure that changes in performance across the plugged period were not caused by increasing familiarity with the task requirements. Subjects were recruited through departmental emails and public notices and received payment for their contribution. All subjects provided informed consent before participation in the study. Ethical approval was provided by the Central University Research Ethics Committee of the University of Oxford.

Earplugs. The earplugs used were the commercially available E.A.R. Classic. Figure 1 shows the mean pure tone thresholds as a function of frequency, both before plugging and on the first day of plugging for all subjects. The audiogram clearly shows that the attenuation caused by the earplug is frequency dependent, ranging from $\sim 23 \mathrm{~dB}$ at $125 \mathrm{~Hz}$ to $\sim 50$ $\mathrm{dB}$ at $8 \mathrm{kHz}$.

Free-field stimuli. Free-field testing was controlled by custom-designed software (written in Delphi 5.5) that generated the stimuli, recorded responses to them, and provided feedback when necessary. Subjects were seated within a circle of 12 loudspeakers, positioned $30^{\circ}$ apart $(100 \mathrm{~cm}$ radius) (Fig. $2 A$ ), in a double-walled sound-attenuating chamber, and
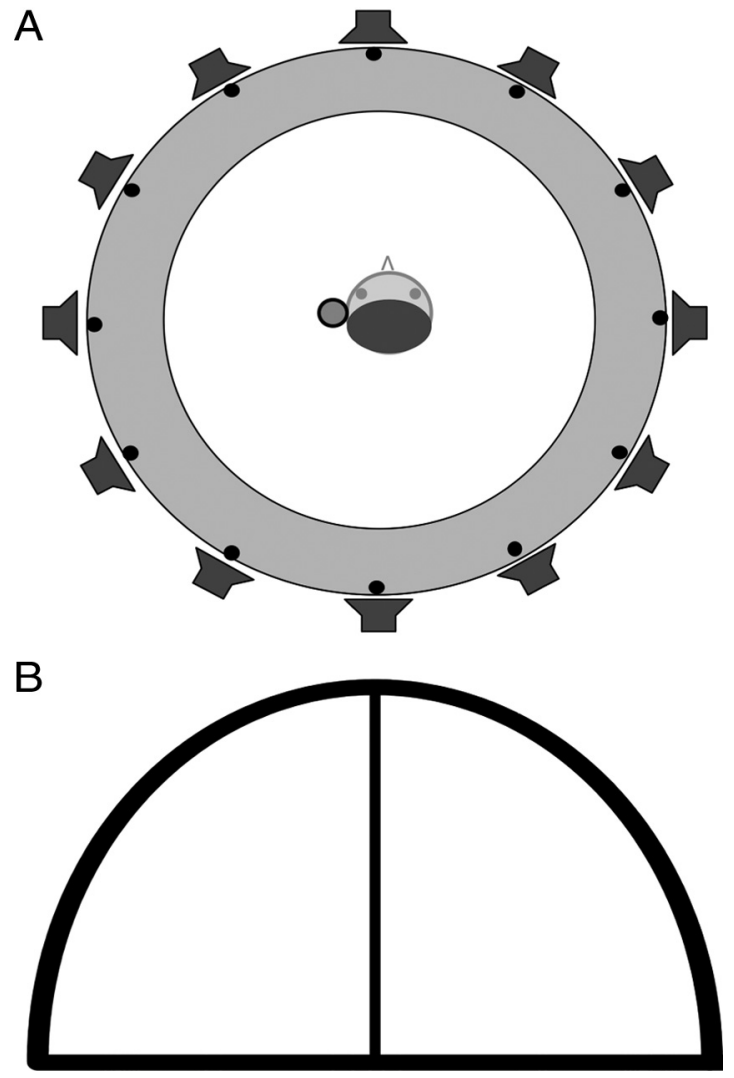

Figure 2. Behavioral testing setups. $A$, Free-field setup. Subjects sat in the middle of a circle of 12 speakers, placed at $30^{\circ}$ intervals. Head movements were restrained during stimulus presentation using a chin rest. $\boldsymbol{B}$, Closed-field setup. Subjects responded to stimuli presented over headphones using a graphical user interface that represented the frontal hemifield of auditory space.

were required to place their chin upon a rest that brought their interaural axis to the same height as the speakers $(105 \mathrm{~cm})$ and also prevented head movements during testing. A graphical user interface (GUI) displayed on a flat-screen monitor represented the positions of the speakers and allowed for subjects to respond by clicking a mouse cursor over the appropriate speaker symbol.

Free-field speaker calibrations were performed by positioning a Brüel and Kjær 4191 condenser microphone where the center of the subject's head would be during the presentation of a stimulus. Golay codes (Zhou et al., 1992) were presented and processed using a Tucker Davis Technologies (TDT) System 3 RP2.1 signal processor and a Brüel and Kjær 3110-003 measuring amplifier. Transfer functions were calculated for each of the 12 speakers. Finite impulse response (FIR) filters were then computed from the inverted Fourier spectra and used to flatten the output of each speaker. The same setup was used to measure the A-weighted sound pressure level from each speaker, and presentation level was adjusted so that it was equivalent for each speaker and appropriate for the stimuli in question.

During testing, stimuli were presented using TDT System 2 hardware. All stimulus durations were $300 \mathrm{~ms}$. For the purposes of this study, we use the term flat spectrum noise to refer to stimuli for which the average spectral output was flattened and matched by the FIR filters from trial to trial and random-filtered noise to refer to stimuli for which the spectral content was further filtered by randomizing the stimulus in $1 / 6$ octave bands by up to $40 \mathrm{~dB}$.

For the flat spectrum noise stimuli, Gaussian noise $(0-20 \mathrm{kHz}, 5 \mathrm{~ms}$ rise-fall time) was generated on each trial using digital-to-analog conversion at $83.333 \mathrm{kHz}$, passed through an anti-aliasing filter (TDT FT5) and a programmable attenuator (TDT PA4), and then amplified using a QUAD 240 power amplifier. A power multiplexer (TDT PM1) was used to direct the stimulus to one of the 12 different speakers (Audax 
TW025M0). On each trial, stimuli were pseudorandomly presented at one of the six sound levels $(50,56,63,70,77$, or $84 \mathrm{dBA})$ to minimize the availability of level cues during the task. The random-filtered stimuli were generated and presented in the same way as the flat spectrum stimuli, with two important differences; after application of the FIR filter to flatten the spectral output of the appropriate speaker, the stimulus intensity was randomly roved over a $40 \mathrm{~dB}$ range within $1 / 6$ octave bands (Wightman and Kistler, 1997). This gave, on any given trial, an unpredictable stimulus spectrum and therefore reduced the availability of spectral cues in the computation of sound-source location. Because of this roving, the mean overall sound intensity on any given trial was held constant at $50 \mathrm{dBA}$, to avoid the presentation of particularly loud stimuli within any specific frequency band.

Closed-field stimuli. Closed-field stimuli were passed to a TDT System 3 RM1 mobile processor for digital-to-analog conversion and presented to subjects over headphones (Sennheiser HD650). To reduce electrical crosstalk induced by the large ILDs presented in this experiment, the cables running to each headphone were separated along their length, and a shielded $6.3 \mathrm{~mm}$ to $3.5 \mathrm{~mm}$ stereo adaptor was used to connect to the mobile processor. This allowed for a maximal ILD of $30 \mathrm{~dB}$ to be presented. Closed-field level calibrations were performed in much the same way as the free-field level calibrations, using a Brüel and Kjær 4134 closed-field condenser microphone and a Brüel and Kjær 4153 artificial ear, which was held in place inside the cup of each headphone during calibration. Because the frequency response of these headphones is extremely flat between $16 \mathrm{~Hz}$ and $30 \mathrm{kHz}$, it was decided not to attempt to flatten their output further.

The closed-field tasks were designed to measure the effects of a unilateral earplug on ILD and ITD lateralization and were also controlled by custom-designed software that had been written using Matlab (r.2007b; MathWorks). The software presented the GUI, generated stimuli, and recorded responses to them. For stimuli containing energy below $\sim 1$ $\mathrm{kHz}$, bone conduction thresholds are reduced by the presence of an earplug (Dirks and Swindeman, 1967), which can lead to perceived amplification of a stimulus presented over headphones (known as the "occlusion effect"), presenting a potential confound for our closed-field lateralization tasks. Changes in ILD lateralization were therefore measured using high-pass Gaussian noise stimuli with a low corner frequency of $1.9 \mathrm{kHz}$, thereby omitting this low-frequency region and specifically targeting the high-frequency region, in which ILD sensitivity is optimal and lateralization using fine-structure ITDs is not possible. To avoid the large, high-frequency ILD introduced by the earplug, we capped the upper frequency range of our ITD noise stimulus at $1.5 \mathrm{kHz}$. The highpass frequency used for the ITD task was determined by measuring ITD sensitivity in the presence of a unilateral earplug for three highly trained subjects using a bandpass filter with low corner frequencies ranging from 0.5 to $1 \mathrm{kHz}$. We used an ITD range that spanned both the physiological range and beyond, from 0 to $1500 \mu$ s favoring the left or right ear. The results were highly consistent across subjects. Figure $3 A$ shows, for one subject, how the percentage of responses to the side of the open ear, taken as a rough measure of left-right response bias, changed depending on the value used for the bottom edge of the bandpass filter. For values $>0.9$ $\mathrm{kHz}$, this bias strongly favors the open ear, indicating that boneconducted ILDs are minimal and that the natural, acoustic ILD introduced by the earplug is dominating lateralization of the ITD stimulus. For the condition in which the stimulus was bandpassed from 0.5 to 1.5 $\mathrm{kHz}$, the occlusion effect pushed the overall response bias toward the plugged ear. For the runs in which the bottom edge of the filter was varied from 0.6 to $0.9 \mathrm{kHz}$, there appeared to be a tradeoff region, in which the bias is relatively stable and, as might be expected, slightly favors the open ear. ITD lateralization was then measured using stimuli with either a 0.5 kHz (Fig. 3B) or a $0.9 \mathrm{kHz}$ (Fig. 3C) bottom corner frequency. These runs, each comprising 130 trials, were performed in the presence of a sufficient ILD offset to "re-center" the ITD stimulus. This ILD offset was obtained by having the subjects perform a plugged ILD lateralization run with the same stimulus and fitting a sigmoidal psychometric function to the data using probit analysis (Finney, 1971). We then extrapolated the amount by which, in decibels, the $50 \%$ point (i.e., the point at which the
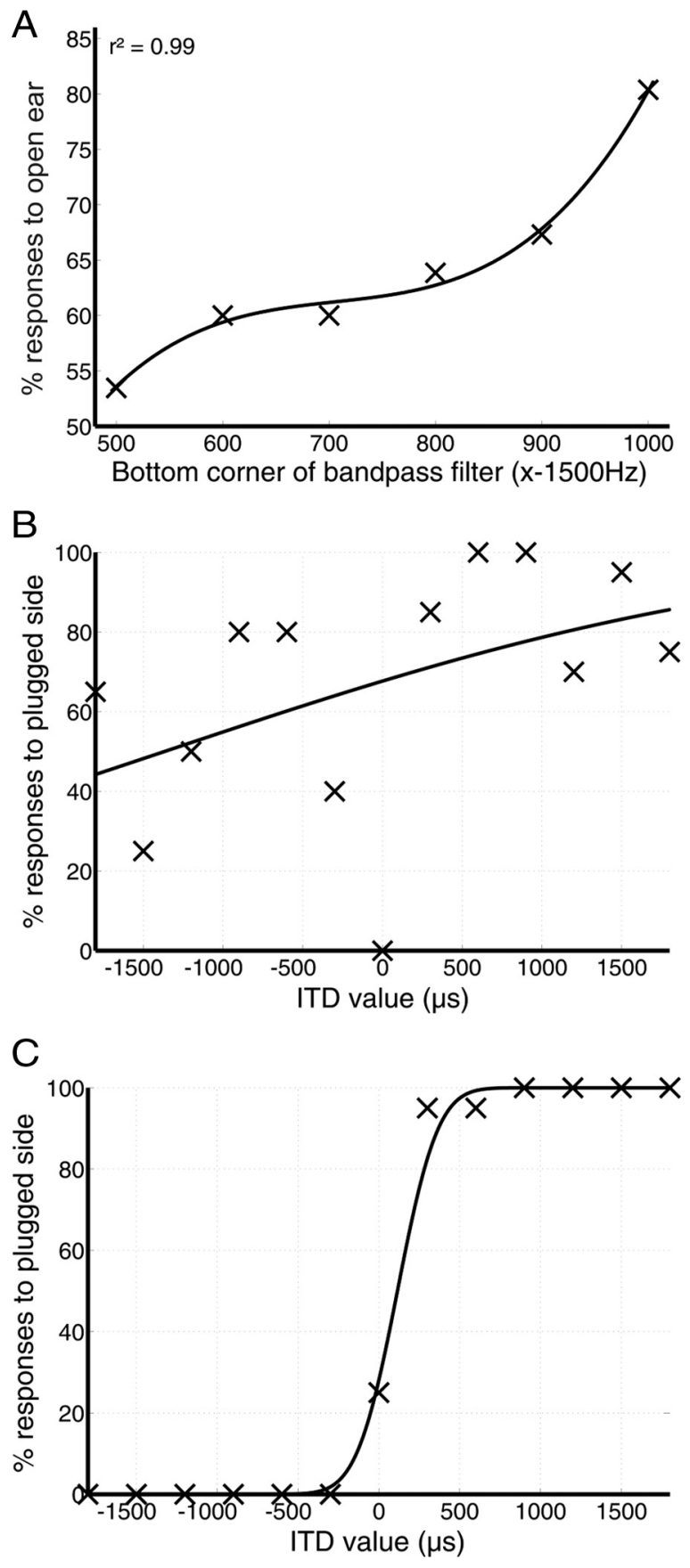

Figure 3. Characterizing the impact of the occlusion effect caused by bone conduction on ITD lateralization. A, Percentage of responses toward the side of the open ear for one subject as a function of the bottom frequency of a bandpass filter applied to Gaussian noise stimuli. At each filter cutoff, ITD values ranged from 0 to $1500 \mu$ sfavoring the left or right ear. Above $900 \mathrm{~Hz}$, the ILD caused by the earplug dominates discrimination and biases responses toward the open ear. At intermediate values, the bias is relatively stable. Below $600 \mathrm{~Hz}$, the subject's responses start to shift toward the side of the plugged ear, indicating that the occlusion effect causes an ILD favoring the plugged ear. $\boldsymbol{B}$, ITD lateralization data obtained for stimuli with a $0.5 \mathrm{kHz}$ bottom corner frequency with an appropriate ILD offset added to counteract the ILD introduced by the earplug. This subject's responses seem to be heavily biased toward the plugged (in this case, right) ear. C, Data taken from the same subject for a $0.9 \mathrm{kHz}$ bottom corner frequency.

subject perceived the stimulus to be at the midline) was pushed away from the midline as a result of the effect of the earplug.

The data shown in Figure $3 B$ are very noisy, with the majority of responses being made to the side of the earplug, regardless of the ITD value presented. However, when the bottom corner of the bandpass filter 
was set at $0.9 \mathrm{kHz}$, the data could be modeled with a sigmoidal psychometric function (Fig. 3C), indicating that ITD lateralization was now possible with this stimulus. It was therefore decided that the stimulus used for ITD lateralization should be bandpassed between 0.9 and 1.5 $\mathrm{kHz}$, using an ILD offset that was determined before each ITD run using the method outlined above. Although this permitted only a relatively narrow pass band of useful ITD information, it did allow us to measure changes in ITD sensitivity across the plugged period that were independent of either the natural or the bone-conducted ILD introduced by the earplug. Subjects were asked to confirm that the offset ILD produced an ITD stimulus that was perceived to be on the midline when the ITD was set at $0 \mu \mathrm{s}$. We also collected one control run (130 trials) in each test session, in which subjects performed the ITD lateralization task without the ILD offset present.

All closed-field stimuli were generated and presented at a sample rate of $48 \mathrm{kHz}$. Cosine ramps of $20 \mathrm{~ms}$ duration were used to ramp the stimulus on and off, and the overall stimulus duration was $300 \mathrm{~ms}$. The average binaural level for all closed-field stimuli was kept constant at $65 \mathrm{dBA}$.

Free-field psychophysical task. Subjects triggered the start of each trial by clicking on a crosshair located at the center of the response GUI (based on Fig. $2 \mathrm{~A}$ ). They were then presented with a stimulus from one of the 12 surrounding speakers. Subjects indicated the speaker location from which they perceived the stimulus to have originated by clicking over one of the 12 speaker symbols on the response GUI.

During the pretraining phase and in the flat spectrum training sessions (see below), feedback was provided in several ways: (1) a colored marker appeared on the GUI over the correct speaker location, along with, if appropriate, a tag indicating the direction and magnitude of the angular error made, and (2) the current overall percentage correct score and the percentage correct score for each speaker location were updated after every trial. Each free-field run consisted of 125 trials.

Closed-field psychophysical task. Subjects started each closed-field run (comprising 130 trials) by clicking anywhere on the arc on the response GUI (Fig. 2B). During pretraining, this was immediately followed by presentation of a series of ILDs or ITDs that swept across the 13 binaural cue values used in the experiment, from $30 \mathrm{~dB}$ or $690 \mu$ s favoring the right side to the same values favoring the left side of the midline and back again. The red arc was split into 13 evenly spaced segments, and, on presentation of each binaural cue value, a yellow bar appeared in the region corresponding to the value presented. At the end of this sweep, which served to familiarize subjects with the relationship between the cue values and the appropriate response locations, the first stimulus was presented and subjects were required to indicate, by clicking the appropriate region on the red arc, where they perceived the stimulus to have originated. Their response was immediately followed by feedback in the form of a yellow bar that appeared over the region corresponding to the cue value that had just been presented. After a pause of $1 \mathrm{~s}$, the next stimulus was presented. During the test sessions, both the initial "sweep" across the binaural range and the trial-by-trial feedback were omitted. Although this response system was arbitrary in the sense that the "locations" to which each ILD or ITD corresponds could vary slightly with head size and therefore between individuals, it did allow us to record a continuous response variable for the range of binaural values used in this experiment. We were therefore able to examine changes in response bias across the whole binaural range from one session to the next.

Pretraining phase. To ensure that any changes seen early in the plugged phase were not attributable to improvements in expertise on any of the tasks or to learning-related effects with particular stimuli, a pretraining phase was provided, comprising 1.5-2 h of testing per day over 5-6 d in the absence of an earplug. During this phase, both experimental and control subjects first performed several runs on the flat spectrum freefield task. Control subjects then started the "plugging" phase, whereas the experimental subjects continued pretraining and moved over to the random-filtered free-field task for several runs. This was followed by training on each of the three closed-field tasks, starting with the $>1.9$ $\mathrm{kHz}$ ILD condition, followed by the $0.9-1.5 \mathrm{kHz}$ ILD condition (for determining the ILD offset), and finishing with the $0.9-1.5 \mathrm{kHz}$ ITD condition. This phase also constituted a screening period, in which sub- jects whose performance did not reach criterion could be excluded. Thus, subjects ( $n=6$ ) who did not attain a score of $\geq 85 \%$ on the flat spectrum free-field task within the first $6 \mathrm{~d}$ were excluded from the plugging phase of the experiment. This ensured that all subjects performed to a high and consistent level before wearing an earplug. Feedback (as described above) was provided for all tasks during the pretraining phase. Subjects were also given extensive training in inserting the earplug during this phase.

Plugging (experimental) phase. For the daily-trained/all group $(n=$ $10)$, the plugging phase consisted of five test sessions $(\sim 2.5 \mathrm{~h}$ each $)$ that were performed in the following sequence: before plug, day 1 of plugging (performed on the same day), day 4 of plugging (mid-test), day 8 of plugging (final day wearing the earplug), and postplug (performed as soon as the earplug was removed). Audiograms were conducted on both ears during the pre-plug and first plugged sessions. Each of the five sessions comprised the following stimulus conditions: free-field flat spectrum noise ( 1 run, 125 trials) and free-field random-filtered noise ( 1 run, 125 trials). In addition, for five of the 10 subjects in this group, the following stimulus conditions were used: closed-field ILD testing with high-pass noise ( 2 runs, 260 trials); closed-field ILD offset characterization with noise bandpassed from 0.9 to $1.5 \mathrm{kHz}$ ( 1 run, 130 trials); closedfield ITD testing with noise bandpassed from 0.9 to $1.5 \mathrm{kHz}$, with a superimposed ILD offset to re-center the stimulus ( 2 runs, 260 trials); and closed-field ITD testing with noise bandpassed from 0.9 to $1.5 \mathrm{kHz}$, with no superimposed ILD offset ( 2 runs, 260 trials). No feedback was provided on any of the tasks during the test sessions. On the intervening days between test sessions, all 10 subjects in this group completed two training runs on the free-field flat spectrum condition (2 runs, 250 trials), with feedback in place.

For the daily-trained/flat subjects $(n=5)$, testing (again without feedback) was performed with the free-field flat spectrum stimuli only before inserting the earplug (pre-plug), on day 1 of plugging (performed on the same day), on the final day of plugging, and after plug removal (postplug, performed on the same day as the final earplug test session). Localization training with free-field flat spectrum stimuli in the presence of feedback ( 125 trials per day) took place on all the other days during which the earplug was in place. The block-trained subjects $(n=5)$ completed $750-800$ trials on the free-field flat spectrum condition in $1 \mathrm{~d}$, taking breaks when necessary.

During the plugged phase, subjects wore the earplugs all day, taking them out only to shower or sleep. Fresh earplugs were inserted every $1-2 \mathrm{~d}$.

Free-field data analysis. Localization performance on our free-field tasks was assessed by calculating the percentages of correct scores and of front-back confusions. We also determined whether unilateral earplugging introduced a lateral bias or change in localization accuracy by first subjecting the stimulus and response azimuths to the following transformation: $\sin$ Azim $\leftarrow \sin ($ Azim $\times 2 \times \pi / 360)$. This removes the impact of front-back errors on the dataset, because, on the transformed scale, a value of 0 could refer to either $0^{\circ}$ or $180^{\circ}$ azimuth (i.e., the posterior and anterior portions of the circular response space were "folded" onto each other, effectively turning all errors into lateral errors). We then obtained the best linear fit to the stimulus-response relationship using a leastsquares fitting algorithm. The calculated model coefficients allowed us to assess measures of left-right bias (the intercept of the fitted function), localization judgments across the stimulus range ("gain," or the slope of the fitted function), and subjects' consistency, or certainty, in their responses (the correlation coefficient of the fitted function). For all factorial analyses conducted on the free-field data, nonsignificant terms were removed from the fitted models until the minimal adequate model had been found, and the reported significance values are from these minimal adequate models. When $t$ tests were conducted, if a marginal $p$ value was obtained, the analysis was confirmed using the nonparametric Wilcoxon's signed-rank test.

Closed-field data analysis. Because of the relatively small number of subjects who completed the closed-field tasks $(n=5)$, group analyses were not performed. Instead, for each subject, we fitted an ANCOVA model with separate slopes and intercepts for each testing session and examined differences between sessions using multiple comparisons with values from the $t$ distribution. We thus tested for significant comparisons 


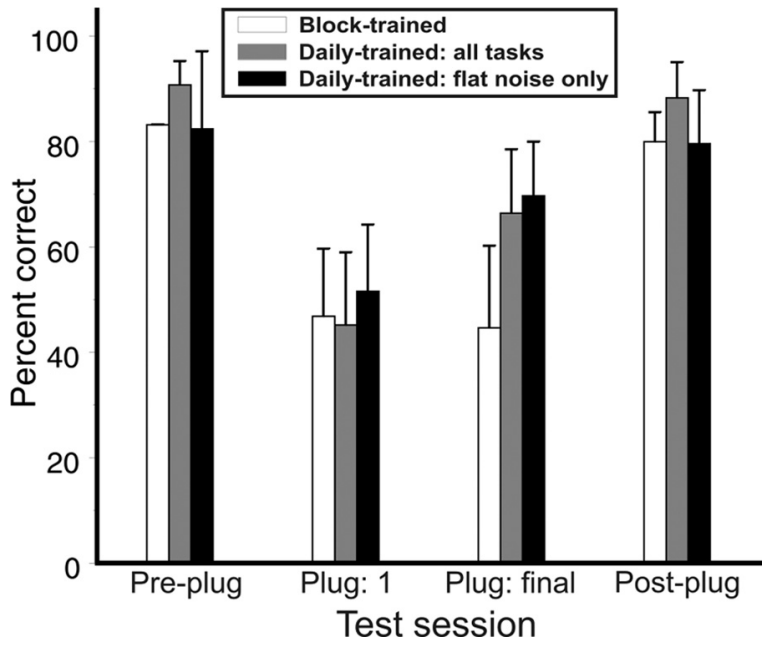

Figure 4. Effects of task exposure on the localization of the flat spectrum stimulus in the presence of a unilateral earplug. Percentage correct scores did not change significantly for subjects who received all their training trials in $1 \mathrm{~d}$ (Block-trained). However, subjects who received daily training did improve, and the extent of that improvements was unaffected by whether they were also tested with the random-filtered stimulus and performed closed-field tasks (Daily-trained: all tasks) or just performed the free-field task with the flat spectrum stimulus (Daily-trained: flat noise only). Error bars are SDs.

at the $95 \%$ confidence level. For the ITD data, it was clear from the pre-plugging sessions that there was already a small amount of bias in the responses to extreme ITD values, with fewer responses being made to the angular values around $90^{\circ}$. Furthermore, the variance across the response range was not always homogenous, and, in some cases, the stimulusresponse relationship was non-monotonic. These factors likely reflect the difficulty of the task, given that we were forced to restrict our stimulus pass band to a narrow frequency range at the upper limit of human ITD lateralization capability to avoid the acoustic confounds introduced by the occlusion effect. In view of this, we only analyzed ITDs in the range of $\pm 346 \mu$ s, to target the range where subjects seemed to be most certain about their lateralization judgments and where the stimulus-response relationship was at its most linear. We then fitted the ANCOVA model as described above. We were thus able to estimate psychophysically the impact of the earplug on each subject's angular response (the intercept, or bias, of the fit), as well as to estimate the delay introduced by the earplug, from the ITD value required for the stimulus to be perceived as originating at the midline.

These analyses were performed on the raw intercepts and slopes obtained in our fitted models. However, for both the ILD and the ITD data, we express absolute response bias in degrees. The slope values are expressed as a proportion of each subject's best value (i.e., closest to the $x=y$ diagonal), allowing for meaningful comparisons of changes in response gain both within and between subjects. Thus, a subject operating at their optimal performance level would have a bias of $0^{\circ}$ and a gain of 1 .

\section{Results}

Free-field performance: effects of daily versus

\section{blocked training}

No differences were found between subjects who were plugged in the left and right ears, so all data reported here were standardized such that negative angular or stimulus values represent the plugged ear and positive values the open ear.

Figure 4 shows the mean percentage correct scores for freefield performance with the flat spectrum noise stimulus (i.e., the training stimulus) for all 20 subjects who took part in this study, subdivided according to how they were tested. The mean scores for all three groups fell to $\sim 50 \%$ correct on the first day of earplugging, and no differences were found between the groups either before plugging (ANOVA; $F_{(2,17)}=2.26, p=0.13$ ) or after insertion of the earplug $\left(F_{(2,17)}=0.39, p=0.68\right)$. To assess whether there were any group differences in the way in which localization performance changed over the period in which the earplug was in place, the absolute change in percentage correct scores across the plugged period was calculated for each subject, and the mean value for each group regressed against plugged session number. The data for the block-trained group, who performed all their trials on the same day, were split into six consecutive bins of $\sim 125$ trials each, to allow for an appropriate comparison with the data from the first six sessions with the flat spectrum stimulus from the other two groups. By the end of session 6 , the change in percentage correct score was $-2 \pm 10.4 \%$ (mean $\pm \mathrm{SD}$ ) in the block-trained group. In contrast, both groups who performed daily training sessions showed an improvement in performance. The mean change in percentage correct was $+17.7 \pm 3.8 \%$ in the daily-trained/flat subjects, who were tested with free-field flat spectrum stimuli only and $+16 \pm$ $8.07 \%$ in the daily-trained/all group. The only group to exhibit a regression slope that was not significantly different from 0 and thus a lack of training-induced improvement was the blocktrained group ( $p=0.94)$. This finding is supported by the presence of a significant interaction between the effect of experimental group and session number (ANCOVA; $F_{(2,114)}=$ 9.33, $p<0.001)$. Examination of the treatment contrasts confirmed that only the block-trained subjects showed a significant difference in slope from either of the other groups (all $p<0.001$ ).

This analysis shows that daily training results in a significant recovery in localization accuracy in humans wearing a unilateral earplug. However, performing a similar total number of trials in $1 \mathrm{~d}$ on an identical localization task did not result in an improvement in performance. There were no differences in the degree of improvement seen in the two groups who were trained daily, and a two-way ANOVA conducted on the absolute change in percentage correct scores for these two groups between the first and last day of plugging indicated no significant effect of experimental group $\left(F_{(1,11)}=0.37, p=0.55\right)$ or of the number of training trials completed during that period $\left(F_{(1,11)}=0.12, p=0.73\right)$. Interestingly, regardless of the changes observed during the plugged period, removal of the earplug resulted in an immediate return to pre-plug performance levels in all subjects (Fig. 4). Because the groups that received daily training showed no differences in rate of improvement with the flat spectrum noise stimulus, we combined the data obtained using this task from these groups for additional analyses.

\section{Free-field performance: effects of sound level}

Figure 5 shows the mean percentage correct scores obtained by the 15 subjects who received daily training with the flat spectrum noise stimulus. The data obtained at each of the six sound levels used are shown before the earplug was inserted, during the plugged phase of the experiment, and after earplug removal. For these subjects, plugging one ear resulted in a drop in performance from $88 \pm 0.09$ to $47.3 \pm 0.13 \%$ correct. Across the plugged period, performance improved steadily, finally reaching a mean percentage correct score of $67.5 \pm 0.11 \%$. The slope of the linear fit to the daily mean percentage correct scores was significantly different from $0\left(t_{(6)}=7.82, p<0.001\right)$, and the scores obtained by each subject on the first and last day of plugging differed significantly $\left(t_{(14)}=-7.46, p<0.0001\right)$. After removal of the earplug, the percentage correct scores were no different from their pre-plugging values $\left(t_{(14)}=1.76, p=0.1\right)$.

To confirm that subjects were not using level cues introduced by the head shadowing effect to perform lateral judgments during 


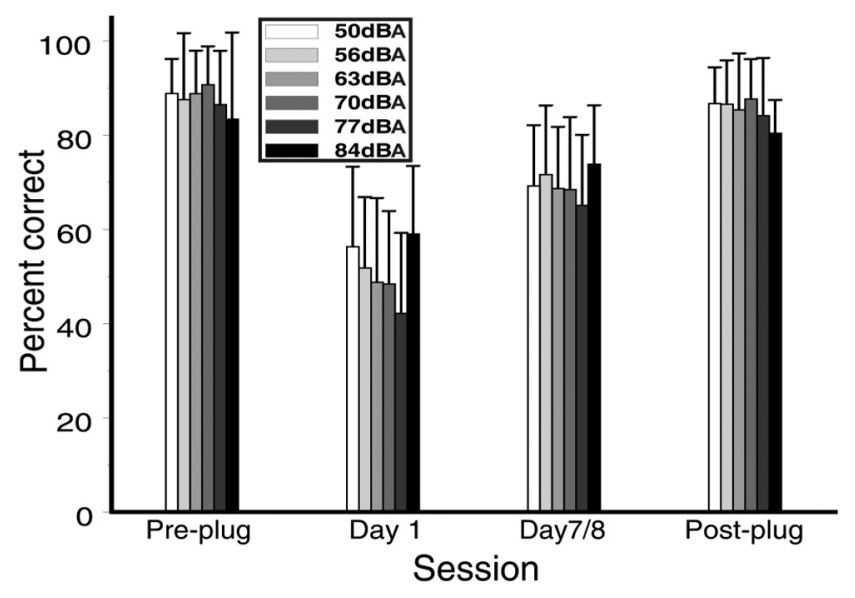

Figure 5. Percentage correct scores for the daily-trained subjects as a function of the level of the flat spectrum stimulus before, during, and after plugging one ear. Error bars are SDs. Performance was similarly disrupted at all levels and improved to a similar degree at all sound levels after 1 week of unilateral earplugging. The percentage correct scores then returned to pre-plug values after removal of the earplug.

the task, we conducted a multiple linear regression on the transformed responses (see Materials and Methods) from the first day of plugging, with stimulus level and response azimuth as the predictors. The predictors were converted to $z$-scores to make them dimensionless. In all but one case, the azimuth coefficients were $>0.8$, and the overall mean value for the azimuth coefficient was $0.88 \pm 0.11$, close to the maximum value of 1 for a perfect relationship between the predictor and the responses. The highest value obtained for the stimulus level coefficients was 0.21 , and the mean value was $0.01 \pm 0.08$, with approximately half of the values obtained being negative. By the final day of plugging, this trend had not changed, with the azimuth coefficients being more tightly clustered around 1 and the sound-level coefficients being more tightly clustered around 0 . These analyses therefore imply that, after insertion of a unilateral earplug, the subjects were not using level judgments at the open ear to perform the task but instead immediately began using whatever localization cues were available to them. For all additional analyses, data were combined across sound level.

\section{Free-field performance: localization changes during the plugged period}

Figure $6 A$ illustrates the pattern of improvement in localization accuracy by the stimulus-response plots taken from an example subject, and Figure $6 B$ shows the biases and gains for each subject at corresponding stages of the experiment. Before insertion of the earplug, this subject was performing almost perfectly, with nearly all responses lying on the $x=y$ diagonal. Plugging one ear led to an increase in front-back errors on both sides (as indicated by the responses falling within the gray boxes), but the proportion of these errors was greater on the side of the plugged ear. There was also an increase in errors that were not front-back confusions. By the end of the plugging period, localization accuracy had improved as the responses again clustered around the diagonal. After earplug removal, the distribution of localization responses closely resembled that seen before earplugging.

The pattern of improvement seen during the plugged period differed on the left and right sides of space. After one ear was plugged, subjects tended to perform better on the side of the open ear. The mean percentage correct scores fell from $85 \pm 0.11$ to $36.7 \pm 0.13 \%$ on the plugged side, rising to $58.9 \pm 0.13 \%$ by the final day of plugging (Fig. 7A). Performance was substantially better on the side of the open ear, with scores falling from $87.9 \pm$ 0.11 to $52.2 \pm 0.19 \%$ after earplugging and recovering to $69.2 \pm$ $0.15 \%$ by the final plugged day. This difference between the two hemifields was significant (ANCOVA; $F_{(1,227)}=24.5, p<$ $0.0001)$, as was the improvement seen on each side $\left(F_{(1,227)}=36\right.$, $p<0.0001)$. Although the subjects were less accurate on the side of the earplug, the rate of improvement across the plugged training sessions was similar to that observed on the side of the open ear $\left(F_{(1,226)}=0.79, p=0.37\right)$.

To investigate further this pattern of improvement, we calculated the percentage of front-back confusions in each hemifield (Fig. $7 B$ ). Plugging one ear resulted in a significant increase in the incidence of front-back errors from $1.3 \pm 3.9$ to $18.2 \pm 7.9 \%$ on the plugged side $(t$ test, $p<0.0001)$ and from $0.22 \pm 0.58$ to $8.34 \pm 8.28 \%$ on the side of the open ear ( $t$ test, $p<0.01$ ). By the final day of plugging, the proportion of front-back errors had declined significantly on both sides ( $t$ tests, $p<0.05$ ) as the overall performance improved. On both the first and last days of the plugging period, significantly more front-back errors were made on the side of the earplug than on the side of the open ear ( $t$ tests, $p<0.01)$.

These data indicate that the training-induced improvement in localization accuracy in the presence of a unilateral earplug can be attributed, at least in part, to a gradual reduction in front-back errors. To investigate the contribution of other factors to the adaptive plasticity, we examined the biases (intercepts), gains (slopes), and $R^{2}$ values for least-square linear fits to the data after they had been transformed to take account of front-back errors. We also calculated the percentage of left-right errors, i.e., responses that were mislocalized to the wrong side of the midline. At pre-plugging, subjects generally performed at near-optimal levels, with biases that were not significantly different from 0 $\left(t_{(14)}=0.77, p=0.45\right)$, slopes that were not significantly different from $1\left(t_{(14)}=1.07, p=0.3\right.$ ), and very high $R^{2}$ values (mean \pm $\mathrm{SD}, 0.99 \pm 0.01)$. The percentage of left-right errors was extremely low during this session $(0.47 \pm 1.22 \%)$. Subjects were thus localizing extremely accurately and with a high degree of certainty in their responses.

After insertion of the earplug, the signed change in left-right response bias across all subjects was not significant $\left(t_{(14)}=\right.$ $-1.21, p=0.24$ ), and the bias values were not significantly different from $0\left(t_{(14)}=1.29, p=0.22\right)$. However, examination of the second panel in Figure $6 B$ suggests that the absolute bias did increase, regardless of direction. Because six listeners were biased toward the side of the earplug in this session, we conducted $t$ tests on both the bias change for those who became biased toward the open ear $\left(t_{(8)}=3.22, p<0.05\right.$; confirmed with signed-rank test, $p<0.01)$ and the pooled unsigned bias change for all listeners $\left(t_{(14)}=4.3, p<0.001\right)$. The implication of these analyses is that, although some listeners naturally became biased toward the open ear because of the perturbation caused by the earplug, others could have reacted by overcompensating with their responses. This is reflected in the fact that the percentage of left-right errors increased significantly in the first plugged session $\left(7.58 \pm 7.67 \% ; t_{(14)}=-3.68, p<0.01\right)$. The gains of the fitted functions $\left(t_{(14)}=3.76, p<0.01\right)$ and their $R^{2}$ values $\left(t_{(14)}=\right.$ $4.17, p<0.001)$ were reduced when the ear was first plugged, although, in all cases, the stimulus-response correlation remained significant (all $p<0.01$ ).

Comparison of the responses obtained on the first and last days of plugging revealed a shift in bias toward the midline that approached significance $\left(t_{(14)}=2.04, p=0.06\right)$ and a significant 
A

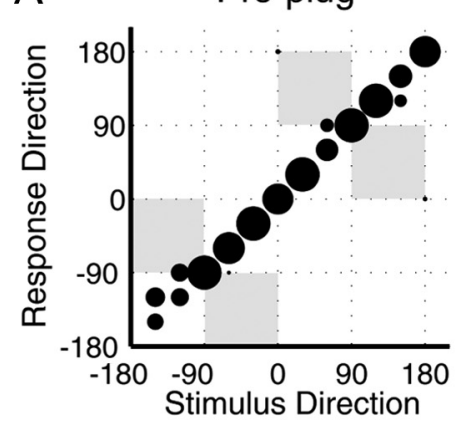

B

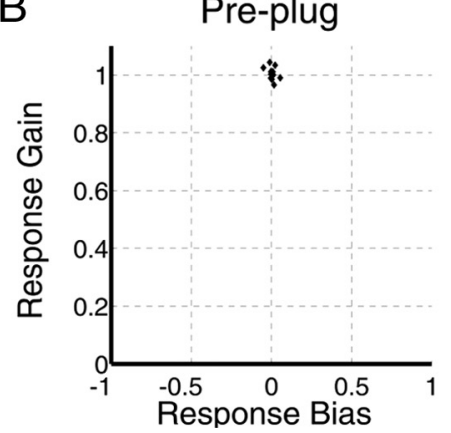

Plug: Day 1

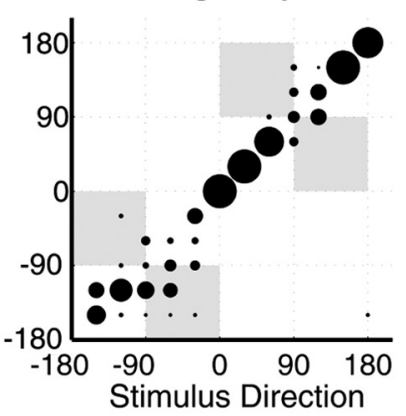

Plug: Day 1

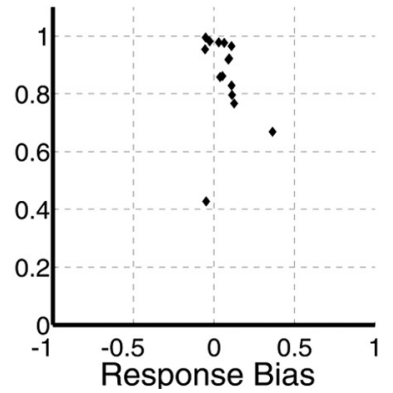

Plug: Day $7 / 8$

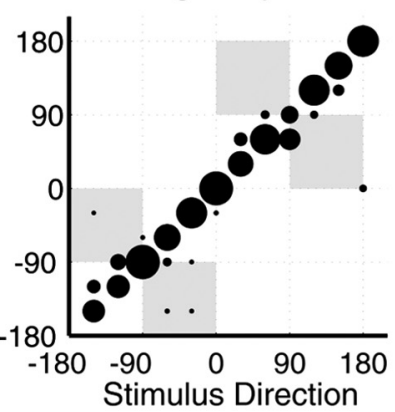

Plug: Day $7 / 8$

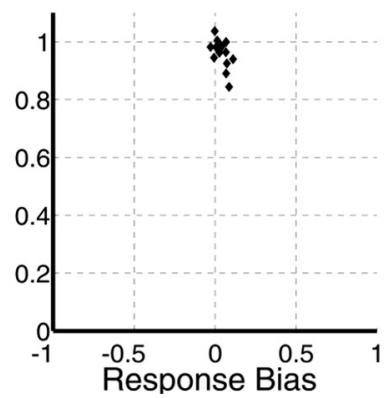

Post-plug

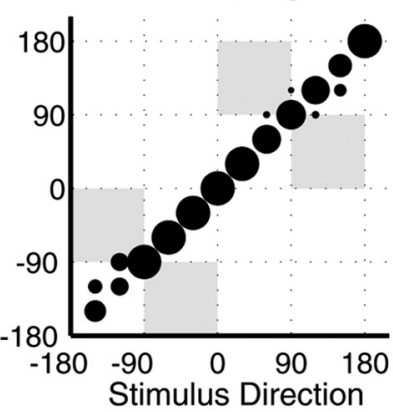

Post-plug

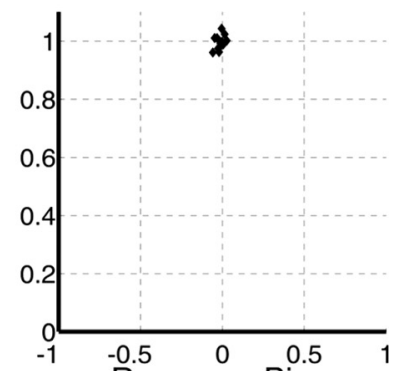

Figure 6. A, Confusion matrices showing, for one subject, changes in the stimulus-response relationship for the flat spectrum stimulus across the plugged period. Negative values represent stimuli presented from the plugged side. Plugging one ear immediately impaired localization accuracy, particularly by increasing the incidence of front- back errors (shaded regions). By the final day of plugging, the number of errors made had declined. Removal of the earplug caused performance to return to pre-plugging levels. $\boldsymbol{B}, 0 \mathrm{bserved}$ gains and biases obtained by fitting linear regressions to the stimulus-response data obtained from each subject. Negative biases represent stimuli presented from the plugged side. Plugging one ear biased the responses toward the side of the open ear and reduced response gains in most subjects. Both measures of localization performance had essentially recovered by the final day of plugging and returned to pre-plugging values after removal of the plug.

reduction in the incidence of left-right errors $(4.34 \pm 4.45 \%$; $t_{(14)}=2.49, p<0.05$; confirmed with signed-rank test, $\left.p<0.05\right)$. The gains of the fitted functions also increased $\left(t_{(14)}=-3.06, p<\right.$ $0.01)$, although they remained significantly different from 1 $\left(t_{(14)}=-3.2, p<0.01\right)$, indicating that sound localization accuracy had not completely returned to pre-plugging levels. The stimulus-response correlation also improved significantly over this period $\left(t_{(14)}=-3.72, p<0.01\right)$, with a final plugging day $R^{2}$ value of $0.96 \pm 0.02$. Comparison of the left and right panels in Figure 6, $A$ and $B$, shows that localization responses after earplug removal closely resembled those recorded before earplug insertion. There was no difference between these two sessions in terms of the $R^{2}$ values $\left(t_{(14)}=1.83, p=0.09\right)$, although there was a small reduction in response gain that approached significance $\left(t_{(14)}=2.12, p=0.05\right)$. However, there did seem to be a small aftereffect of plugging on the left-right response bias, with postplugging values shifted toward the side of the previously plugged ear $t_{(14)}=2.22, p<0.05$; confirmed with signed-rank test, $p<$ $0.05)$. These post-plug bias values were also significantly different from $0\left(t_{(14)}=-2.26, p<0.05\right.$; confirmed with signed-rank test, $p<0.05)$.

\section{Free-field performance: effects of disrupting the} stimulus spectrum

For the 10 subjects in the daily-trained/all group, pre-plugging performance with the random-filtered noise stimulus (mean \pm SD, $89.4 \pm 0.04 \%$ ) (Fig. $8 A$ ) was comparable with that for the flat spectrum noise stimulus $\left(91 \pm 0.04 \% ; t_{(9)}=1.03, p=0.33\right)$. However, the subjects' ability to localize random-filtered noise was severely disrupted after plugging one ear, particularly on the side of the earplug (Fig. $8 \mathrm{~B}$ ), and the percentage correct scores obtained were significantly lower than with the flat noise $\left(t_{(9)}=4\right.$, $p<0.01$ ). This was partly attributable to an increase in frontback errors, which rose from $1.9 \pm 1.6 \%$ in the pre-plug session to $18.3 \pm 7.5 \%$ on the first day of plugging $\left(t_{(9)}=6.11, p<\right.$ 0.001 ), although the subjects also made a large number of left-right errors. In contrast to the substantial recovery in performance observed with flat noise, there was no change in percentage correct scores $\left(t_{(9)}=-1.49, p=0.17\right)$ or the proportion of front-back errors $\left(t_{(9)}=0.67, p=0.52\right)$ between the first and final days of plugging, suggesting that learning is prevented when spectral cues are unreliable (Fig. $8 A, B$ ). In support of this, a two-way ANOVA revealed a significant interaction between testing session and stimulus type $\left(F_{(4,86)}=8.1, p<0.0001\right)$, indicating that localization accuracy changed in different ways for the two types of stimulus over the course of the plugging period. After removal of the earplug, the subjects' percentage correct scores achieved with the random-filtered noise immediately returned to preplugging levels $\left(t_{(9)}=0.4, p=0.69\right.$ ) (Fig. $8 A, B$ ), as did the proportion of front-back errors $\left(t_{(9)}=0.29, p=0.78\right)$.

Figure $8 C$ shows the effect of 1 week of unilateral earplugging on the left-right bias and the gain of the localization responses. In the pre-plug test with random-filtered noise, response bias was not significantly different from $0\left(t_{(9)}=1.49, p=0.17\right)$, gains were not significantly different from $1\left(t_{(9)}=-0.04, p=0.97\right)$, and the mean $R^{2}$ value was $0.99 \pm 0.003$. Accordingly, the percentage of left-right errors was extremely low $(0.18 \pm 0.38 \%)$. When one ear was plugged, responses became biased to the openear hemifield $\left(t_{(9)}=-4.74, p<0.01\right)$, consistent with the lower percentage correct scores on the side of the earplug $\left(t_{(9)}=6.11\right.$, $p<0.001)$ and with the significant increase in percentage of left-right errors $\left(21.75 \pm 13.38 \% ; t_{(9)}=-5.07, p<0.0001\right)$. The 


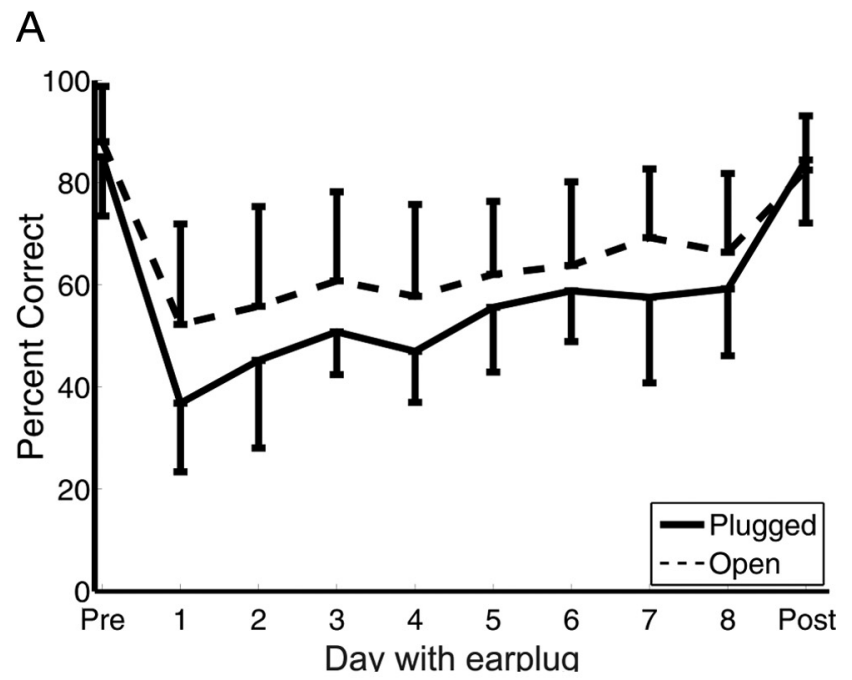

B

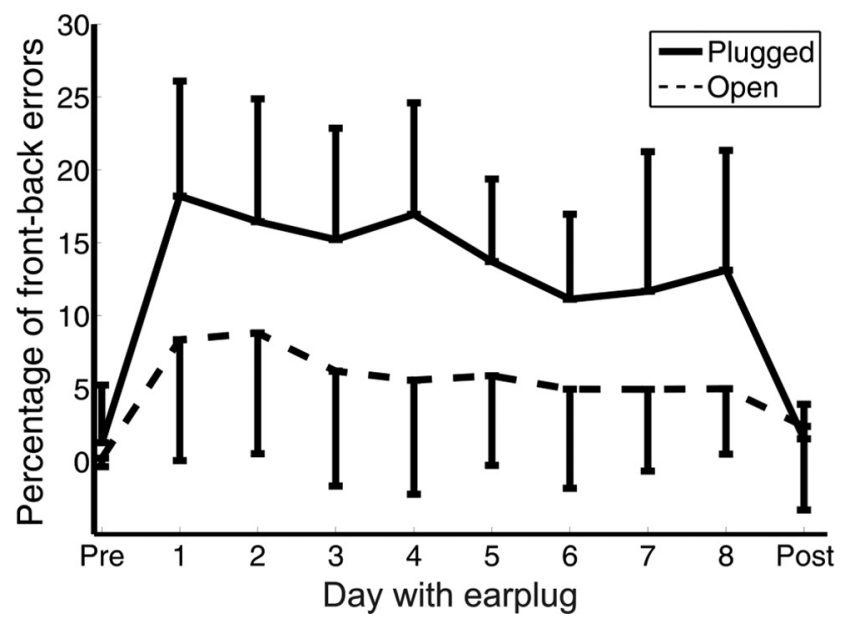

Figure 7. Localization performance of the daily-trained subjects for flat spectrum noise stimuli before the earplug was inserted (Pre), on each of the days on which the ear was plugged, and after removing the earplug (Post). Data are shown separately for the side of the plugged ear (Plugged) and the open ear (Open). A, Percentage correct scores. $\boldsymbol{B}$, Percentage of front-back errors. Error bars are SDs. Although performance was degraded more severely on the side of space ipsilateral to the earplug, a similar improvement was observed on both sides.

gains of the fitted functions were also significantly reduced for all subjects $\left(t_{(9)}=4.85, p<0.001\right)$, and the $R^{2}$ values dropped to $0.68 \pm 0.21$. Nevertheless, the $R^{2}$ values remained significant for all subjects $(p<0.01)$, indicating that the stimulus-response relationship was preserved to some degree. This pattern of results persisted across the plugged period, with none of these measures changing significantly in value ( $t$ tests for comparison of the first and final days of plugging; $p>0.05$ ), confirming the lack of plasticity shown in Figure $8, A$ and $B$. As soon as a balanced binaural input was restored, the subjects were once again able to localize random-filtered noise with great accuracy, as indicated by a return of the gains to values that differed from neither preplug values $\left(t_{(9)}=1.13, p=0.29\right)$ nor $1\left(t_{(9)}=-0.98, p=0.35\right)$ and of the $R^{2}$ values $(0.99 \pm 0.006)$ to those measured before earplugging. However, as with the flat spectrum noise stimulus, there was a small aftereffect of plugging on the left-right response bias $\left(t_{(9)}=2.27, p<0.05\right)$.

\section{Closed-field performance: ILD lateralization during the plugged period}

Figure $9 A$ shows example data from one subject before, during, and after the plugged period, and Figure $9 B$ shows the fitted response biases and gains for all five subjects across the same period. The data from the pre-plug session revealed that four subjects had response gains close to 1 and left-right biases close to $0^{\circ}$, whereas the fifth subject lateralized the ILDs less well and seemed to have an inherent response bias toward one side. Nevertheless, the overall mean bias for all five subjects was small $\left(2.91 \pm 3.85^{\circ}\right)$. In every subject, insertion of an earplug produced a significant reduction in gain (mean gain, $0.59 \pm 0.12$ ) and biased the responses significantly toward the side of the open ear (mean bias, $38.8 \pm 7.04^{\circ}$ ). Indeed, the subject whose data are shown in Figure $9 A$ made virtually no responses to the side of the earplug. By the final day of plugging, one subject had developed a significantly smaller bias, and one had a bias that was significantly larger. The remaining three subjects showed no change in response bias across the plugged period. Two subjects showed significant improvements in gain, one showed a significant reduction, and the others did not change. Given the lack of a clear trend for change on these measures during the plugged period, these data do not provide conclusive evidence for ILD plasticity across the plugged period. This is supported by the fact that both the mean gains $(0.63 \pm 0.13)$ and biases $\left(36.5 \pm 0.08^{\circ}\right)$ were virtually unchanged from those obtained on the first day of plugging. Removal of the earplug resulted in response gains $(0.95 \pm 0.02)$ that were not significantly different from their high pre-plug values for four subjects, whereas for the remaining subject, the gain actually improved. However, there was some indication of an aftereffect of 1 week of earplugging on the measured biases, with four subjects' responses significantly favoring the side of the previously plugged ear (mean bias, $-6.23 \pm 5.15^{\circ}$ ).

\section{Closed-field performance: ITD lateralization during the plugged period}

Figure $10, A$ and $B$, shows the data obtained from an example subject in the ITD lateralization task. When the individually measured offset ILD was omitted from the ITD (bandpassed) stimulus (Fig. $10 \mathrm{~B}$ ), the responses were heavily biased toward the side of the open ear, indicating that the ILD introduced by the earplug was dominating the lateralization percept. Adding an appropriate ILD offset, as described in Materials and Methods, brought the subject's responses back toward the midline and improved the stimulus-response relationship (Fig. 10A). Figure 10C shows that, before plugging, the gain for four of the five subjects was very high, in three cases having a value of 1 (mean gain, $0.94 \pm$ 0.12 ), and all subjects displayed left-right response biases that were close to $0^{\circ}$ (mean bias, $0.4 \pm 2.7^{\circ}$ ). Thus, their perceived midline was relatively central. Insertion of the earplug caused a significant drop in gain for two subjects, but in the rest, the gain did not change significantly. Four subjects experienced a significant shift in response bias toward the side of the open ear, indicating that the earplug had delayed the signal in the plugged ear. The mean shift in response bias was $16.5 \pm 12.1^{\circ}$, and this equated to a mean delay, taking each subject's pre-plugging bias values into account, of $147.3 \pm 109 \mu \mathrm{s}$. Between the first and last days of earplugging, no significant changes in ITD lateralization occurred, and only one subject showed a significant shift in response bias. Similarly, for most subjects, no differences were seen in the gain or bias between the pre-plug and post-plug sessions, although the responses of two subjects were shifted significantly 

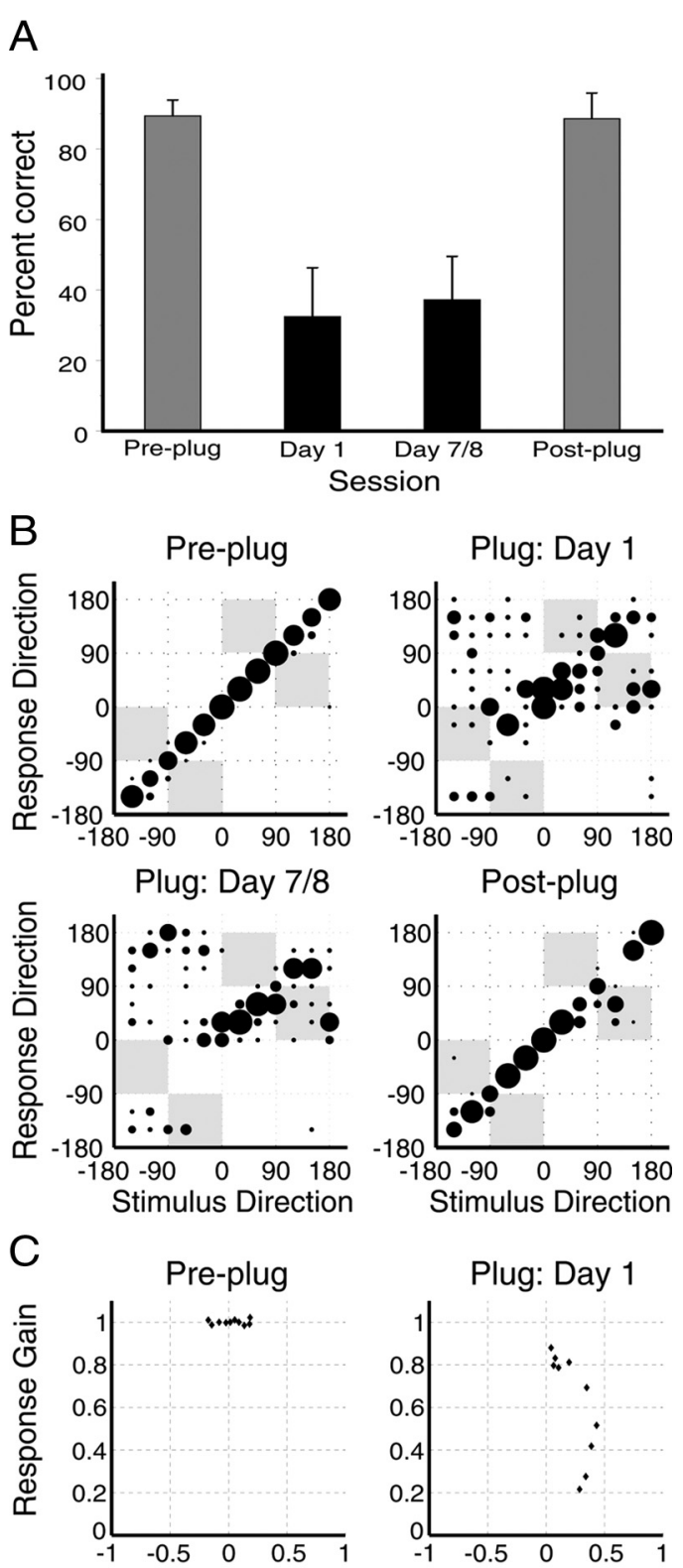

Plug: Day $7 / 8$
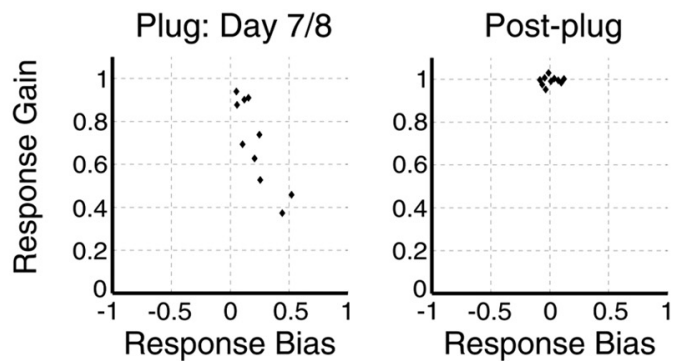

Figure 8. Impact of unilateral earplugging on free-field sound localization with a randomfiltered noise stimulus. $\boldsymbol{A}$, Mean percentage correct scores before, during, and after the plugged period. Insertion of an earplug caused severe disruption of sound localization that had not recovered by the end of plugging (black bars), but removal of the plug caused a return to pre-plugging levels (gray bars). Error bars are SDs. $\boldsymbol{B}$, Confusion matrices showing changes in the stimulus-response relationship across the plugged period for the subject depicted in Figure $6 \mathrm{~A}$. Negative values represent stimuli presented from the plugged side. Insertion of an earplug disrupted sound localization performance, producing an increase in front-back errors and in responses to the side of the open ear. This pattern had not recovered by the end of plugging. Post-plug performance closely resembled that seen before insertion of an earplug. C, Observed gains and biases obtained by fitting linear regressions to the stimulus-response data obtained from each subject. Jitter has been applied to the bias values in the top left and bottom right toward the side of the previously plugged ear and one exhibited a lower gain.

Given that there did not seem to be any ITD adaptation to the earplug over the course of the plugged period in this study, we decided to use all the data from the plugged period (plug day 1 , mid-test, and final day of plugging) from each subject to estimate more accurately the delay introduced by an earplug. For these data, the mean change in the ITD value required to produce a predicted response at the midline was very close to the value we obtained when we used only the data from the first day of plugging $(149.9 \pm 81.96 \mu \mathrm{s})$, indicating that, although there was substantial variability in the means obtained from person to person, the trend was for a delay value that remained extremely constant.

\section{Discussion}

We investigated the characteristics of sound localization plasticity in humans after altering the spatial cues available by chronically plugging one ear. Simple block exposure to the training task was not sufficient to produce improvements in performance, whereas daily training led to a progressive recovery in sound localization accuracy. Adaptive plasticity was observed, however, only for broadband stimuli with a flattened, and therefore predictable, amplitude spectrum and not for stimuli whose spectral composition was randomized from trial to trial. Together with the lack of evidence for ILD or ITD adaptation and the very small aftereffects observed on all tasks when the earplug was removed, these results indicate that the process of learning to accommodate degraded inputs in one ear involves a reweighting of the different localization cues.

In keeping with the results of related studies (Bauer et al., 1966; Butler, 1987), we found that the horizontal localization abilities of all subjects who received daily sound localization training progressively shifted back toward their pre-plug performance after several days of unilateral earplugging. The extent of the recovery closely matched that reported for monaurally plugged ferrets trained to localize broadband sound in a comparable 12-speaker setup (Kacelnik et al., 2006). In that study, the degree of plasticity exhibited by the ferrets was determined by the frequency of training, implying that additional training might lead to more complete adaptation to the earplug in humans.

Human listeners can be rapidly trained to reinterpret the relationship between auditory localization cues and directions in space (Shinn-Cunningham et al., 1998). Our results suggest that a longer period of training is required, however, to compensate for a reduced input in one ear. The lack of improvement observed after plugging one ear in subjects who performed all trials with the "training" stimulus in $1 \mathrm{~d}$ is consistent with other learning studies in humans showing that improvements in performance occur between, rather than within, daily training sessions (Karni and Sagi, 1993; Wright and Sabin, 2007). It also seems likely that the opportunity for the subjects to interact with their surroundings while wearing the earplugs outside the psychophysical setup, and perhaps to benefit from feedback provided by vision and self-generated movements, may have contributed to the recovery in localization accuracy.

$\leftarrow$

panels for presentation purposes. Negative values represent stimuli presented from the plugged side. Plugging one ear biased the responses toward the side of the open ear and reduced response gains in most subjects, with both changes persisting until the final day of plugging. Gains recovered when the plug was removed, although we did see some evidence for a bias toward the previously plugged ear. 

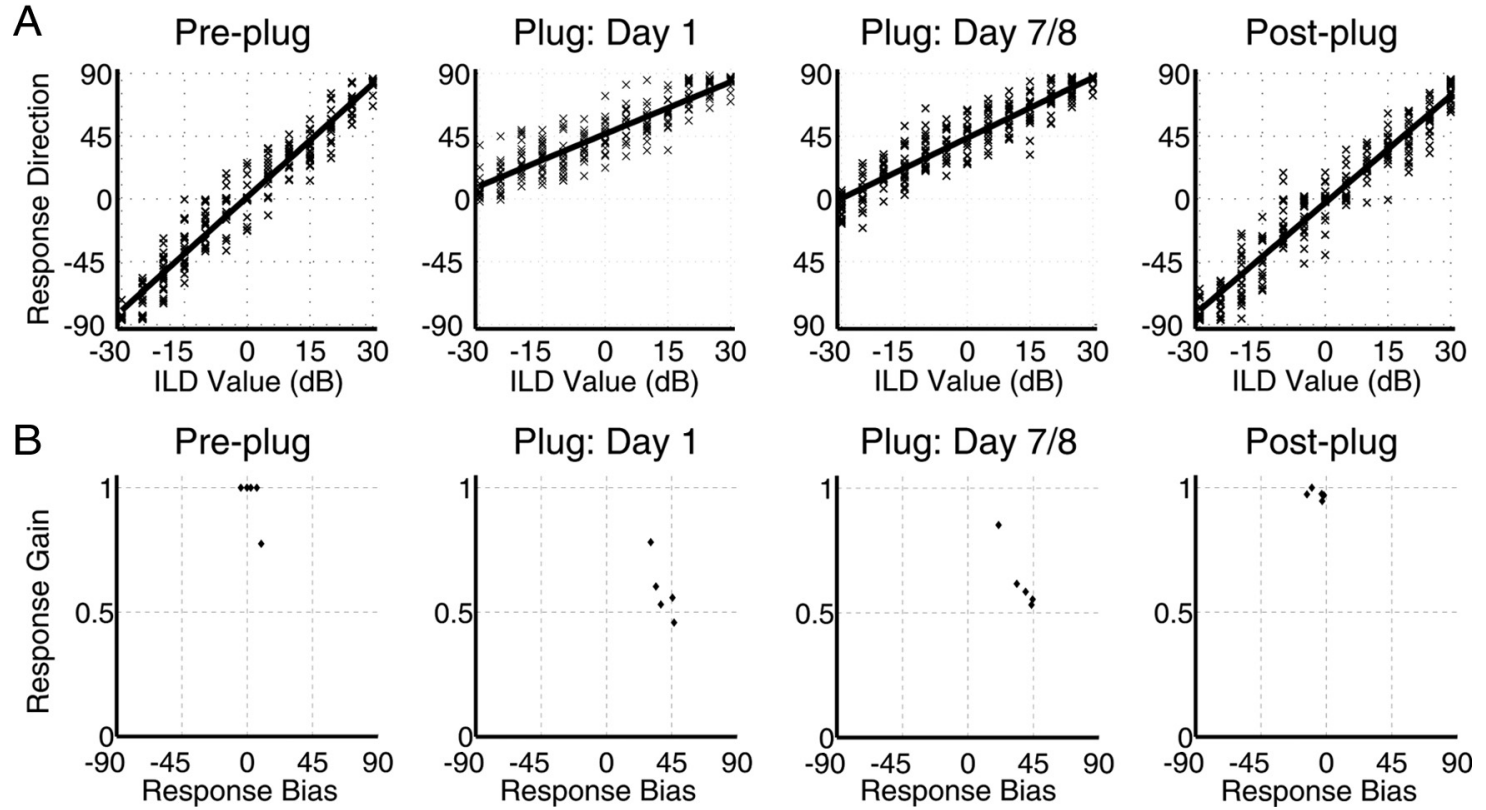

Figure 9. Impact of unilateral earplugging on closed-field ILD lateralization. $\boldsymbol{A}$, Linear fits to the stimulus-response data obtained from one subject. Negative values represent stimuli presented from the plugged side. Plugging one ear biases response toward the side of the open ear, a pattern that appears to persist after 1 week of wearing the plug. Removal of the plug causes an immediate return to pre-plug performance. $\boldsymbol{B}, 0$ bserved gains and biases obtained by fitting an ANCOVA model to the stimulus-response data obtained from each subject. Jitter has been applied to the bias values in the leftmost panel for presentation purposes. Negative biases represent stimuli presented from the plugged side. In all subjects, plugging one ear reduced the response gain and increased the bias toward the side of the open ear. Although gains recovered after plug removal, there was some tendency for subjects to respond toward the side of the previously plugged ear.

\section{Acoustical basis for plasticity}

Plugging one ear reduces the level of auditory input and delays the sound at that ear and consequently alters both ILD and ITD values corresponding to particular directions in space. We characterized the attenuation produced by the earplugs from the change in audiometric thresholds and quantified the delay psychophysically from the change in left-right bias on the ITD task. The values obtained are consistent with those reported after electrophysiological measurements in monaurally plugged animals (Moore et al., 1999; Hartley and Moore, 2003).

Adaptation to a unilateral earplug could be achieved by altering neuronal sensitivity to one or other of these binaural cues, as has been demonstrated in developing barn owls (Mogdans and Knudsen, 1992; Gold and Knudsen, 2000). Perceptual learning studies in adult humans have also highlighted the potential for plasticity in the processing of ILDs (Wright and Fitzgerald, 2001; Kumpik et al., 2009; Zhang and Wright, 2009) and, to a lesser extent, ITDs (Rowan and Lutman, 2006). Moreover, imposition of altered binaural cues can lead to adaptive adjustments in cue sensitivity. For example, Javer and Schwarz (1995) found that sound localization accuracy gradually recovered after introducing a delay in one ear via a hearing aid, implying plasticity in ITD processing. Moreover, a previous study with earplugs reported that the ILD required for dichotically presented low-frequency tones to be centered in the head changed after several days of unilateral earplugging (Florentine, 1976), although McPartland et al. (1997) were only able to partially replicate this result.

In contrast to these reports of plasticity in binaural cue sensitivity, our findings provide little support for either ILD or ITD adaptation. However, the use of broadband noise with a consistent source spectrum as a free-field training stimulus provided our listeners with access to monaural spectral cues as well as ILDs and ITDs. Along with the binaural cues, monaural spectral cues would have been severely disrupted for the plugged ear but essentially unchanged for the open ear. On the basis of the very small aftereffect observed after restoring normal binaural inputs in ferrets that had adapted to an earplug, we proposed that auditory spatial learning involves a shift in sensitivity away from the abnormal cues to those cues that are less affected by the earplug (Kacelnik et al., 2006). In particular, the spectral cues provided by the open ear appear to be critical for this, because modifying those cues by reversibly reshaping the open external ear of ferrets that had adapted to chronic plugging of the other ear immediately reduced their localization accuracy to the level observed when the earplug was first inserted.

The results of the present study provide additional support for cue reweighting as the basis for plasticity. First, we observed a similarly modest aftereffect of plugging in the data obtained from human subjects in both free-field and closed-field tasks. Although indicating that the previously plugged ear was contributing to their localization judgments, this aftereffect appears too small to be consistent with any substantial adaptation to the altered cues, a conclusion supported by the lack of systematic changes in ILD or ITD lateralization as learning occurred on the free-field task. Second, we found that localization of flat spectrum noise recovered with training, whereas no improvement occurred for broadband stimuli in which spectral cues were disrupted by introducing trial-to-trial fluctuations in the source spectrum. Third, part of the improvement in performance with training was attributable to a reduction in the incidence of front-back errors, which are resolved on the basis of spectral cues (Oldfield and Parker, 1984; Carlile and King, 1994). Together, these results strongly suggest that recovery of accurate localization in the horizontal plane in the presence of a unilateral earplug is associated with a greater reliance on spectral cues. Although this reweighting presumably takes place gradually as performance improves with training, our findings support the conclusion drawn by Van Wanrooij and Van Opstal (2007) that disruption of binaural cues leads to an immediate dependence on the intact spectral cues provided by the open ear. This is because 

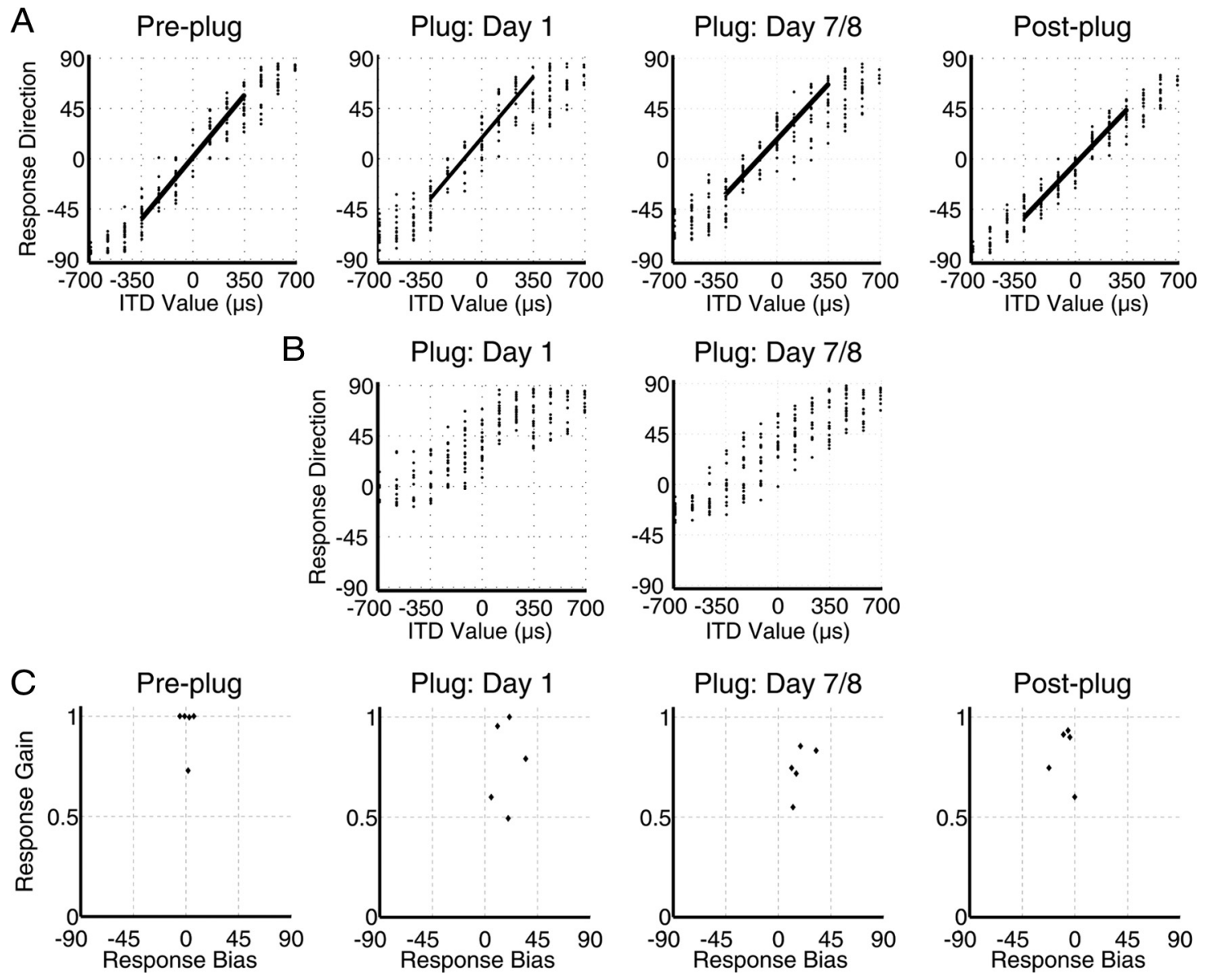

Figure 10. Impact of unilateral earplugging on closed-field ITD lateralization. $A$, Linear fits to the stimulus-response data obtained from one subject in the presence of an appropriate ILD offset. Negative values represent stimuli presented from the plugged side. Insertion of an earplug causes a bias shift toward the side of the open ear that remained throughout the plugged period. $\boldsymbol{B}$, ITD lateralization without an adequate ILD offset present in the stimulus; the acoustic ILD introduced by the plug dominates the perception of lateralized stimuli, and the responses are mostly on the side of the open ear. C, Observed gains and biases obtained by fitting an ANCOVA model to the stimulus-response data obtained from each subject when an appropriate ILD offset was present in the stimulus. Jitter has been applied to the bias values in the leftmost panel for presentation purposes. Insertion of an earplug induces a significant bias shift toward the unplugged ear in most subjects and a significant reduction in gain in two of them. There is little change in either measure throughout the plugged period.

localization accuracy on the first day of plugging was significantly worse with random-filtered noise than with flat noise, whereas this was not the case before unilateral earplugging.

The finding that recovery of accurate sound localization in the presence of abnormal binaural inputs is based primarily on the greater use of spectral cues is consistent with previous work showing that the human auditory system can learn to use novel spectral cues produced by perturbing the shape of the external ear for sound localization in the vertical plane (Hofman et al., 1998; Van Wanrooij and Van Opstal, 2005). Those studies suggest that plasticity in the processing of spectral cues takes place independently for each ear, supporting the possibility that the intact cues provided by the open ear dominated the localization performance of our earplugged subjects. Nevertheless, we found that percentage correct scores increased and front-back errors decreased by a similar degree in both hemifields, implying that our subjects were still using binaural inputs. Given that Van Wanrooij and Van Opstal (2004) showed that fully monaural listeners localize poorly and use monaural level judgments arising from head shadowing to pinpoint low-intensity sounds (an effect we did not observe), it seems likely that some input through the plugged ear was required for the localization plasticity that we observed. That listeners wearing unilateral earplugs may use interaural local- ization cues is also supported by virtual acoustic space experiments (Wightman and Kistler, 1997).

\section{Physiological basis for plasticity}

Because adaptation to a unilateral earplug appears to involve learning to make greater use of spectral-shape information while ignoring abnormal binaural cues, it seems likely that plasticity takes place at a processing stage where these cues come together. Recording studies have shown that adaptive adjustments in auditory spatial tuning take place in the optic tectum/superior colliculus of barn owls (Knudsen, 1985) and ferrets (King et al., 1988) that were raised with one ear plugged. However, more relevant to our results is the finding that elimination of cortical neurons that project to the inferior colliculus (IC) prevents adult ferrets from adapting to an earplug (Bajo et al., 2010) and that reversible inactivation of the cortex produces immediate shifts in the sensitivity of IC neurons to ILDs (Nakamoto et al., 2008). Because IC neurons encode both binaural and spectral cues (Chase and Young, 2008), it is possible that the corticocollicular projection brings about experience-driven changes in localization abilities by adjusting the relative sensitivity of the midbrain neurons to those cues.

The finding that the relative contributions of different spatial cues to sound localization can change according to how salient or 
reliable they are would appear to be a particularly effective way of maintaining a stable spatial percept as the availability of the cues changes in different acoustic environments. This may be a more general mechanism of adaptive plasticity, because other studies have shown that optimal multisensory integration can involve a change in the weighting afforded to different sensory cues according to how reliable they are (Alais and Burr, 2004; Ronsse et al., 2009). The dynamic coding of auditory space also highlights the potential for recovery of function after a loss of hearing in one ear. In a similar vein, it should be possible to use behavioral training to enhance the auditory localization abilities of individuals whose hearing has been restored via a hearing aid or cochlear implant.

\section{References}

Alais D, Burr D (2004) The ventriloquist effect results from near-optimal bimodal integration. Curr Biol 14:257-262.

Bajo VM, Nodal FR, Moore DR, King AJ (2010) The descending corticocollicular pathway mediates learning-induced auditory plasticity. Nat Neurosci 13:253-260.

Bauer RW, Matuzsa JL, Blackmer F, Glucksberg S (1966) Noise localization after unilateral attenuation. J Acoust Soc Am 40:441-444.

Blauert J (1997) Spatial hearing: the psychophysics of human sound localization. Cambridge MA: Massachusetts Institute of Technology.

Butler RA (1987) An analysis of the monaural displacement of sound in space. Percept Psychophys 41:1-7.

Campbell RA, King AJ, Nodal FR, Schnupp JW, Carlile S, Doubell TP (2008) Virtual adult ears reveal the roles of acoustical factors and experience in auditory space map development. J Neurosci 28:11557-11570.

Carlile S, King AJ (1994) Monaural and binaural spectrum level cues in the ferret: acoustics and the neural representation of auditory space. J Neurophysiol 71:785-801.

Chase SM, Young ED (2008) Cues for sound localization are encoded in multiple aspects of spike trains in the inferior colliculus. J Neurophysiol 99:1672-1682.

Clifton RK, Gwiazda J, Bauer JA, Clarkson MG, Held RM (1988) Growth in head size during infancy: implications for sound localization. Dev Psychol 24:477-483.

Dirks D, Swindeman JG (1967) The variability of occluded and unoccluded bone-conduction thresholds. J Speech Hear Res 2:232-249.

Finney DJ (1971) Probit analysis. Cambridge, UK: Cambridge UP.

Florentine M (1976) Relation between lateralization and loudness in asymmetrical hearing losses. J Am Audiol Soc 1:243-251.

Gold JI, Knudsen EI (2000) Abnormal auditory experience induces frequency-specific adjustments in unit tuning for binaural localization cues in the optic tectum of juvenile owls. J Neurosci 20:862-877.

Hartley DE, Moore DR (2003) Effects of conductive hearing loss on temporal aspects of sound transmission through the ear. Hear Res 177:53-60.

Held R (1955) Shifts in binaural localization after prolonged exposures to atypical combinations of stimuli. Am J Psychol 68:526-548.

Hofman PM, Van Riswick JG, Van Opstal AJ (1998) Relearning sound localization with new ears. Nat Neurosci 1:417-421.

Hofman PM, Vlaming MS, Termeer PJ, Van Opstal AJ (2002) A method to induce swapped binaural hearing. J Neurosci Methods 113:167-179.

Javer AR, Schwarz DW (1995) Plasticity in human directional hearing. J Otolaryngol 24:111-117.

Kacelnik O, Nodal FR, Parsons CH, King AJ (2006) Training-induced plasticity of auditory localization in adult mammals. PLoS Biol 4:e71.

Karni A, Sagi D (1993) The time course of learning a visual skill. Nature $365: 250-252$.

King AJ, Hutchings ME, Moore DR, Blakemore C (1988) Developmental plasticity in the visual and auditory representations in the mammalian superior colliculus. Nature 332:73-76.

King AJ, Parsons CH, Moore DR (2000) Plasticity in the neural coding of auditory space in the mammalian brain. Proc Natl Acad Sci U S A 97:11821-11828.

King AJ, Schnupp JW, Doubell TP (2001) The shape of ears to come: dynamic coding of auditory space. Trends Cogn Sci 5:261-270.
Knudsen EI (1985) Experience alters the spatial tuning of auditory units in the optic tectum during a sensitive period in the barn owl. J Neurosci 5:3094-3109.

Knudsen EI, Esterly SD, Knudsen PF (1984) Monaural occlusion alters sound localization during a sensitive period in the barn owl. J Neurosci 4:1001-1011.

Kumpik D, Ting J, Campbell RA, Schnupp JW, King AJ (2009) Specificity of binaural perceptual learning for amplitude modulated tones: a comparison of two training methods. J Acoust Soc Am 125:2221-2232.

McPartland JL, Culling JF, Moore DR (1997) Changes in lateralization and loudness judgements during one week of unilateral ear plugging. Hear Res 113:165-172.

Middlebrooks JC (1999) Virtual localization improved by scaling nonindividualized external-ear transfer functions in frequency. J Acoust Soc Am 106:1493-1510.

Middlebrooks JC, Green DM (1990) Directional dependence of interaural envelope delays. J Acoust Soc Am 87:2149-2162.

Mogdans J, Knudsen EI (1992) Adaptive adjustment of unit tuning to sound localization cues in response to monaural occlusion in developing owl optic tectum. J Neurosci 12:3473-3484.

Moore DR, Hine JE, Jiang ZD, Matsuda H, Parsons CH, King AJ (1999) Conductive hearing loss produces a reversible binaural hearing impairment. J Neurosci 19:8704-8711.

Nakamoto KT, Jones SJ, Palmer AR (2008) Descending projections from auditory cortex modulate sensitivity in the midbrain to cues for spatial position. J Neurophysiol 99:2347-2356.

Oldfield SR, Parker SP (1984) Acuity of sound localisation: a topography of auditory space. II. Pinna cues absent. Perception 13:601-617.

Ronsse R, Miall RC, Swinnen SP (2009) Multisensory integration in dynamical behaviors: maximum likelihood estimation across bimanual skill learning. J Neurosci 29:8419-8428.

Rowan D, Lutman ME (2006) Learning to discriminate interaural time differences: an exploratory study with amplitude-modulated stimuli. Int J Audiol 45:513-520.

Schnupp JW, Booth J, King AJ (2003) Modeling individual differences in ferret external ear transfer functions. J Acoust Soc Am 113:2021-2030.

Shaw EA, Teranishi R (1968) Sound pressure generated in an external ear replica and real human ears by a nearby point source. J Acoust Soc Am 44:240-249.

Shinn-Cunningham BG, Durlach NI, Held RM (1998) Adapting to supernormal auditory localization cues. I. Bias and resolution. J Acoust Soc Am 103:3656-3666.

Van Wanrooij MM, Van Opstal AJ (2004) Contribution of head shadow and pinna cues to chronic monaural sound localization. J Neurosci 24:4163-4171.

Van Wanrooij MM, Van Opstal AJ (2005) Relearning sound localization with a new ear. J Neurosci 25:5413-5424.

Van Wanrooij MM, Van Opstal AJ (2007) Sound localization under perturbed binaural hearing. J Neurophysiol 97:715-726.

Wenzel EM, Arruda M, Kistler DJ, Wightman FL (1993) Localization using nonindividualized head-related transfer functions. J Acoust Soc Am 94:111-123.

Wightman FL, Kistler DJ (1993) Sound localization. In: Human psychophysics (Yost WA, Popper AN, Fay RR, eds), pp 155-192. New York: Springer.

Wightman FL, Kistler DJ (1997) Monaural sound localization revisited. J Acoust Soc Am 101:1050-1063.

Wright BA, Fitzgerald MB (2001) Different patterns of human discrimination learning for two interaural cues to sound-source location. Proc Natl Acad Sci U S A 98:12307-12312.

Wright BA, Sabin AT (2007) Perceptual learning: how much daily training is enough? Exp Brain Res 180:727-736.

Xu L, Middlebrooks JC (2000) Individual differences in external-ear transfer functions of cats. J Acoust Soc Am 107:1451-1459.

Zhang Y, Wright BA (2009) An influence of amplitude modulation on interaural level difference processing suggested by learning patterns of human adults. J Acoust Soc Am 126:1349-1358.

Zhou B, Green DM, Middlebrooks JC (1992) Characterization of external ear impulse responses using Golay codes. J Acoust Soc Am 92:1169-1171. 